\title{
Keratoconus: A Review of Medical and Surgical Options
}

\author{
Cole M Milliken, David Baxley, Joseph Luvisi, James Landreneau and Karolinne M Rocha \\ Storm Eye Institute, Medical University of South Carolina, Charleston, SC, USA
}

$\mathrm{K}$ eratoconus is a progressive non-inflammatory corneal ectasia characterized by paracentral steepening and thinning. Previous management of this condition involved corneal transplantation, which carried a high rate of complications. Newer procedural echnologies have demonstrated success in halting progression and yielding quality visual outcomes. This article reviews current therapeutic options for patients suffering from keratoconus. Using PubMed and MEDLINE, a comprehensive search was performed to identify literature relating to currently available treatments for keratoconus. The literature search revealed that there are multiple effective therapies for keratoconus. These interventions include procedures designed to prevent future ectasia as well as to reduce refractive error by flattening abnormally steep keratometry values. This article includes a discussion of rigid gas permeable lenses, corneal crosslinking, intrastromal implants, combined procedures and corneal transplantation. Novel treatment methods for keratoconus continue to develop as researchers learn more about this ectatic condition. The procedural options depend on the experience of the performing surgeon and appropriate patient selection. Through correct application of recent technologies, many patients have gained meaningful visual improvement without undergoing corneal transplantation.

\section{Keywords}

Keratoconus, corneal crosslinking, corneal transplantation

Disclosures: Cole M Milliken, David Baxley, Joseph Luvisi, James Landreneau and Karolinne M Rocha have no financial or non-financial relationships or activities to declare in relation to this article.

Review process: Double-blind peer review.

Compliance with ethics: This study involves a review of the literature and did not involve any studies with human or animal subjects performed by any of the authors.

Data availability: Data sharing is not applicable to this article as no datasets were generated or analysed during the writing of this article.

Authorship: The named authors meet the International Committee of Medical Journal Editors (ICMJE) criteria

for authorship of this manuscript, take responsibility

for the integrity of the work as a whole, and have

given final approval for the version to be published.

Access: This article is freely accessible at

touchOPHTHALMOLOGY.com (c) Touch Medical Media 2021

Received: 27 October 2021

Accepted: 24 November 2021

Published online: 14 December 2021

Citation: touchREVIEWS in Ophthalmology. 2021;15(2): 46-9

Corresponding author: Cole M Milliken, Storm Eye Institute, Medical University of South Carolina, 71 Ashley Avenue, Charleston, SC 29425, USA. E: millikec@musc.edu

Support: No funding was received in

the publication of this article.
Keratoconus is the most common primary progressive ectatic condition of the cornea. It is classically described as non-inflammatory, distinguishing it from several other pathologies associated with corneal thinning. Prevalence of this disease varies depending on geographical location, with reports as high as $2.3 \%$ in Central India to $0.3 \%$ in Russia. ${ }^{1,2}$ Keratoconus typically presents as a bilateral condition although approximately $14 \%$ of patients may demonstrate unilateral disease. ${ }^{3}$ Keratoconus occurs in both males and females, typically around the second decade of life. The aetiology behind the progressive corneal thinning and steepening is not completely understood; however, independent risk factors include a positive family history, eye rubbing, asthma, allergy and eczema. ${ }^{4}$ Ocular findings and self-reported symptoms depend on severity of progression. Early in the disease course, patients present with significant refractive error due to asymmetric astigmatism and progressive myopic shift. Left untreated, individuals may develop breaks in Bowman's membrane, tears in Descemet's membrane and ultimately severe stromal scarring. ${ }^{5} \mathrm{As}$ our understanding and detection of keratoconus advances, so do the treatment and management options for this disease. This article aims to outline both traditional and novel therapeutic options for keratoconus.

PubMed and MEDLINE were searched for peer-reviewed literature on treatment of keratoconus. search criteria included keywords of 'keratoconus treatment', 'corneal crosslinking' and 'intracorneal ring segments'. The following discussion describes the current treatments available, including some therapies that are not currently approved by the US Food and Drug Administration (FDA) and are strictly off label for the treatment of keratoconus.

\section{Rigid gas permeable and scleral contact lenses}

One of the least invasive options for patients with progressive keratoconus is the fitting of rigid gas permeable and scleral contact lenses. Rigid gas permeable (RGP) contact lenses allow the cornea to adopt a spherical shape due to tear collection behind the lens. The combination of the rigid lens and tear collection behind the lens functions to neutralize existing irregularity from the ectasia while correcting for the patient's underlying refractive error. The primary complaint associated with RGP lenses is discomfort, at which point practitioners can offer scleral lens fitting. ${ }^{\circ}$ Scleral lenses contain a rigid centre, with a softer periphery leading to improved patient tolerability. A 2020 randomized controlled trial reported significantly better comfort ratings for patients wearing scleral lenses compared with RGP lenses.? There does not appear to be any significant difference in visual acuity between the two options. ${ }^{7}$ Additionally, a 2016 retrospective case series in Canada determined the scleral lens to be an acceptable alternative for those who cannot tolerate RGP lenses. ${ }^{8}$ 


\section{Epithelium off corneal crosslinking}

corneal collagen crosslinking $(C X L)$ is currently the treatment of choice for progressive keratoconus. The goal of $\mathrm{CXL}$ is to strengthen corneal stromal fibres in an effort to stabilize the ectatic disease and mitigate further progression. The procedure combines topical riboflavin (vitamin B2) drops and ultraviolet light (UV-A) to strengthen collagen fibres in the cornea. Upon exposure to UV-A light, a photochemical reaction causes the release of reactive oxygen species, resulting in the formation of covalent bonds between collagen and proteoglycan fibres. ${ }^{9}$ This reaction is similar to the molecular changes that occur with the natural ageing process of the cornea. It should be noted that $\mathrm{CXL}$ is not recommended in a stable disease state, but rather in cases of progressive disease or when there is ectasia present after refractive surgery. The most common iteration of the procedure is epithelium-off (epi-off) $\mathrm{CXL}$, during which the corneal epithelium is mechanically removed before topical $0.1 \%$ riboflavin is applied to the surface at intervals of 2-5 minutes for 30 minutes. Following application of the riboflavin, the surface is irradiated with UV-A, inducing the photochemical reaction as previously described. Patients are typically given topical antibiotics and steroids after the procedure, followed by a brief regimen of steroids after re-epithelialization occurs. This technique is called the Dresden protocol, established by Dr Seiler et al. after introducing the concept of CXL in 2003..$^{10}$ Some pitfalls to epi-off CXL include slowed corneal re-epithelialization, which leads to heightened infection risk. In addition, patients often report significant discomfort after the procedure, which can limit its utility in children. A soft bandage contact lens is often applied after the procedure to promote healing of the epithelium. Newer studies demonstrate a matrix therapy agent (ReGeneraTing Agent, RGTA) to promote faster corneal healing and thus a decreased risk of infection, with additional relief in postoperative discomfort. ${ }^{11}$ Finally, damage to the corneal endothelium upon exposure to UV-A light has been reported, particularly in patients with corneal thickness below 400 microns at the time of exposure to UV-A application. Given the progressive corneal thinning that occurs with keratoconus, a large portion of the population fall into the aforementioned category and are not acceptable candidates for CXL. ${ }^{12}$

\section{Epithelium on corneal crosslinking}

Due to unfavourable side effects of epi-off $C X L$, there has been newer interest in epithelium-on (epi-on) or transepithelial CXL. The procedure follows similar principles to epi-off $\mathrm{CXL}$; however, as indicated in the name, the corneal epithelium remains intact. The challenge with epi-on CXL is ensuring that topical riboflavin penetrates to the stromal layers. This issue has been addressed by a variety of methods, including alteration of the concentration of riboflavin, partial micro-puncture of the epithelium, and iontophoresis, which utilizes an electrical gradient to drive riboflavin molecules across the corneal endothelium. ${ }^{13}$ A 2016 systematic review reported that patients receiving epi-on CXL demonstrated a statistically significant improvement in uncorrected distance visual acuity (UDVA), while there was no significant change in Kmax at 3-12 months' followup. An additional systematic review comparing epi-off versus epi-on CXL found no significant difference in UDVA and corrected distance visual acuity (CDVA) at 1-year follow-up between the groups. ${ }^{14} \mathrm{~A}$ primary limitation in evaluating outcomes in epi-on CXL is that there is no single protocol currently utilized by each provider, leading to poor repeatability. Additionally, epi-on CXL has not at this time been cleared for FDA approval in the USA; therefore, its use is strictly off label.

\section{Accelerated crosslinking}

Many investigators have pursued alterations to the Dresden protocol to further optimize surgical management of keratoconus. One such technique is accelerated $C X L$, which aims to maintain the same efficacy as traditional $\mathrm{CXL}$, with the benefits of a shorter procedure. ${ }^{15}$ Similarly to epi-on CXL, several iterations of accelerated crosslinking exist. The accelerated timeline involves increasing UV-A illumination during the procedure, thereby decreasing UV-A exposure time. A prospective interventional case series investigated high-fluence pulsed UV-A at $30 \mathrm{~mW} / \mathrm{cm}^{2}$ for 4 minutes and studied Kmax values at 24 months as the primary outcome. Accelerated pulsed CXL was shown to be safe and effective, with refractively neutral intervention for mitigating disease progression. ${ }^{16}$ As previously mentioned, several methods for delivery and illumination levels have been studied, without universal acceptance of a single approach. One current protocol that is being studied extensively is the use of supplemental oxygen therapy during accelerated crosslinking. ${ }^{17}$ In one of the pathways of corneal crosslinking, singlet oxygen species are generated when excited riboflavin reacts with oxygen. In accelerated crosslinking, there is evidence to support that an alternate low-oxygen reaction pathway is activated more quickly, resulting in decreased efficacy of crosslinking treatment. ${ }^{18}$ Ongoing debate exists on whether accelerated crosslinking protocols, with or without oxygen supplementation, offer comparable or superior efficacy when compared with conventional crosslinking therapy.

\section{Customized crosslinking}

Newer techniques using topography-guided customized corneal crosslinking (PIXL) have provided a more targeted area of treatment to the focal areas of biomechanical weakening in the ectatic cornea. PiXL has shown efficacy in reducing higher-order aberrations, improving best spectacle-corrected distance visual acuity (BSCVA) and hastening epithelial healing compared with standard, generalized crosslinking treatment. ${ }^{19}$ In PiXL, there is debate on whether to apply treatment to the highest keratometry value, thinnest pachymetry point or posterior float. Seiler et al. described the posterior float as the most reliable parameter for targeted treatment, arguing that pachymetry and highest keratometry are both modulated by epithelial thickness. ${ }^{20}$ PiXL has shown promising results for patients with keratoconus, as well as in patients with myopic or astigmatic refractive error. Larger sample sizes and long-term data collection are needed to determine the efficacy of this procedure compared with standard CXL.

\section{Intracorneal ring segments}

The principle of intracorneal ring segments (ICRS) was initially adopted for treatment of severe degrees of myopia. Placement of the segments involves insertion of synthetic semicircular inserts into the mid-peripheral corneal stroma. Pressure exerted by the intrastromal rings creates a shortening of the central arc length, resulting in central corneal flattening as well as forward displacement of the peripheral cornea. ${ }^{21}$ The desired outcome is a decrease in irregular astigmatism, which can improve visual acuity and allow for better refractive correction. A prospective case series evaluating intracorneal ring technology demonstrated a reduction in both astigmatism and spherical correction, as well as an increase in topographical regularity in keratoconus patients. ${ }^{22}$ Additionally, placement of ICRS resulted in improvement of BSCVA in approximately 50\% of patients. ${ }^{23}$ Common complications associated with placement of the synthetic rings include segment migration, externalization of segment, localized inflammatory reaction, patient intolerance and, in rare cases, overlying stromal melt. Overall, the procedure is well tolerated and can be an effective tool in addressing severe corneal steepening with irregular astigmatism.

\section{Combined intracorneal ring segments and corneal crosslinking}

Corneal CXL and ICRS can be used in combination to address severe ectatic disease. When used in combination, $\mathrm{CXL}$ works to mitigate the 
progression of the ectatic cornea and provides biomechanical stability, while ICRS placement works to flatten the cornea and promote topographic regularity. A prospective study suggested that CXL with simultaneous ICRS implantation gives an additional improvement in UDVA with significant reduction in cylinder and spherical equivalent. ${ }^{24}$ Debate still exists as to which procedure should be performed first, with an additional option of performing the procedures simultaneously. A 2018 meta-analysis reviewing best corrected visual acuity (BCVA) and uncorrected visual acuity (UCVA) outcomes for those patients undergoing subsequent (CXL then ICRS) versus simultaneous procedures found superior refractive outcomes in the patient group undergoing simultaneous procedure. ${ }^{25}$ Hersh et al. studied the safety concerns and outcomes of simultaneous ICRS and CXL versus ICRS followed by CXL 3 months later. ${ }^{26}$ Their research showed comparable topographic flattening of the central cone with concurrent ICRS and CXL versus sequential ICRS with CXL. In addition, the side effect profile of combined CXL and ICRS implantation was comparable to that of the respective stand-alone procedures.

\section{Bowman layer inlay transplantation}

An additional surgical procedure for progressive keratoconus is transplantation of an isolated Bowman layer graft. Patient selection for this procedure would include patients with advanced progressive keratoconus who are no longer eligible for CXL but still have adequate BCVA. ${ }^{27}$ The Bowman layer corneal inlay can be inserted via a mechanical tunnel or with femtosecond laser application. Van der Star et al. studied the long-term outcomes of 35 eyes that had undergone Bowman layer inlay transplantation and found stabilization of the corneal curvature in 31 eyes and stabilization of best corrected vision at the 8-year postoperative time point. ${ }^{28}$ In patients with inadequate stromal thickness, there can be difficulty forming a safe tunnel for the Bowman layer transplant. Bowman layer inlay transplantation can offer an alternative surgical approach to deep anterior lamellar keratoplasty (DALK) and photorefractive keratectomy (PRK) in patients with advanced keratoconus.

\section{Autologous stem cell transplantation}

One of the more novel therapies for keratoconus involves implantation of autologous adipose-derived adult stem cells (ADASC) into the corneal stroma. The goal of ADASC implantation is to re-engineer existing tissue of the cornea and to provide an alternative to corneal graft procedures. By inserting stem cells into the scaffolding of the corneal stroma, the cells differentiate into keratocytes and produce new collagen, providing a solution to the underlying pathophysiology of keratoconus..$^{29} \mathrm{~A}$ phase I clinical study evaluating the safety and efficacy of ADASC implantation showed no complications and full corneal transparency after 24 hours in all five patients. ${ }^{30}$ All patients included in the study demonstrated improved visual outcomes, while manifest refraction and topographic keratometry remained stable. This technique offers a promising therapy; however, larger investigative clinical studies need to be conducted before implementation into clinical practice..$^{30}$

\section{Photorefractive keratectomy}

PRK can often be performed in conjunction with CXL to assist in correcting residual refractive error in progressive keratoconus. A 2018 case series showed significant gains in CDVA with no compromise in CXL efficacy over a 24-month follow-up in patients who underwent PRKCXL. ${ }^{31}$ Additionally, a prospective study demonstrated an improvement in mean UCVA and BCVA with better vision quality after simultaneous topography-guided PRK followed by CXL. ${ }^{32}$

Many patients with keratoconus are ineligible to undergo PRK due to severe stromal thinning over the corneal cone. In these cases, transepithelial phototherapeutic keratectomy (t-PTK) can be performed, a procedure that requires less stromal tissue ablation. The combination of CXL and t-PTK is known as the Cretan protocol. A 2019 comparative interventional case series compared the previously mentioned Dresden protocol with the cretan protocol during CXL, and found significant and faster improvements in refractive and keratometric values in the Cretan group. ${ }^{33}$ Further investigation has been performed into refractive surgical techniques for those with mild or forme fruste keratoconus. A 2007 prospective study evaluated outcomes of photoastigmatic refractive keratectomy in patients with average keratometry values less than 50.00 diopters. ${ }^{34}$ The results demonstrated that photoastigmatic correction using the Alpins vector planning technique offered safe and effective myopia and astigmatism correction without sacrificing corneal stability.

\section{Corneal transplantation}

For patients with severe disease that is refractory to previous procedural therapies, often the only remaining option is corneal transplantation. Two of the most popular options for corneal transplantation include full-thickness penetrating keratoplasty (PK) and partial-thickness DALK. DALK involves transplantation of the corneal stroma while leaving intact the host Descemet's membrane and corneal endothelium. A 2019 meta-analysis comparing the two techniques found no difference in BCVA or corneal topography; however, the PK group had a higher rate of transplant rejection and larger residual postoperative spherical equivalent. ${ }^{35}$ Although DALK is associated with a lower rejection and re-transplantation rate, any concurrent endothelial pathology, such as full-thickness corneal scarring, may preclude DALK as a surgical option. Both primary PK and DALK are useful procedures in the treatment of end-stage keratoconus.

\section{Conclusion}

Keratoconus is a commonly encountered disease among ophthalmologists, with numerous therapeutic options available for patients. Regardless of ophthalmic practice, understanding treatment options can benefit patients. Current therapies as discussed above include contact lenses, CXL, ICRS, laser ablation, stem cell transplantation and corneal graft transplantation. Currently, no single perfect management plan exists; rather, each therapeutic plan must be tailored to the patient and to the comfort of the provider. As advances in anterior segment techniques progress, so do the treatment options for keratoconus. The current therapeutic options reviewed in this article aim to improve treatments for patients suffering from this visionthreatening corneal disease. $\square$
. Jonas JB, Nangia V, Matin A, et al. Prevalence and associations of keratoconus in rural Maharashtra in central India: the Centra India Eye and Medical Study. Am J Ophthalmol. 2009;148:760-5

2. Gorskova EN, Sevost'ianov EN. [Epidemiology of keratoconus in the Urals]. Vestn Oftalmol. 1998;114:38-40.

3. Amsler M. Some data on the problem of keratoconus. Bull Sot Belge Ophthalmol. 1961;129:331-54.

4. Hashemi H, Heydarian S, Hooshmand E, et al. The prevalence and risk factors for keratoconus: a systematic review and meta-analysis. Cornea. 2020;39:263-70.

5. Romero-Jiménez M, Santodomingo-Rubido J, Wolffsohn JS,
Keratoconus: a review Cont Lens Anterior Eye 2010:33:157-66. 6. Nau AC. A comparison of synergeyes versus traditional rigid gas permeable lens designs for patients with irregular corneas. Eye Contact Lens. 2008;34:198-200.

7. Levit A, Benwell M, Evans BJW. Randomised controlled trial of corneal vs. scleral rigid gas permeable contact lenses for keratoconus and other ectatic corneal disorders. Cont Lens Anterior Eye. 2020;43:543-52.

8. Yan P, Kapasi M, Conlon R, et al. Patient comfort and visual outcomes of mini-scleral contact lenses. Can J Ophthalmol. 2017;52:69-73.
9. Beshtawi IM, O'Donnell C, Radhakrishnan H. Biomechanical properties of corneal tissue after ultraviolet-A-riboflavin crosslinking. J Cataract Refract Surg. 2013;39:451-62.

10. Wollensak G, Spoerl E, Seiler T. Riboflavin/ultraviolet-a-induced collagen crosslinking for the treatment of keratoconus. Am J Ophthalmol. 2003;135:620-7.

11. Gumus K, Guerra MG, de Melo Marques SH, et al. A new matrix therapy agent for faster corneal healing and less ocular discomfort following epi-off accelerated corneal cross-linking in progressive keratoconus. J Refract Surg. 2017;33:163-70.

12. Wollensak $\mathrm{G}$, Iomdina $\mathrm{E}$. Biomechanical and histological 
changes after corneal crosslinking with and without epithelia debridement. J Cataract Refract Surg. 2009;35:540-6.

13. O'Brart DPS. Corneal collagen crosslinking for corneal ectasias: a review. Eur J Ophthalmol. 2017;27:253-69.

14. Wen D, Li Q, Song B, et al. Comparison of standard versus accelerated corneal collagen cross-linking for keratoconus: a meta-analysis. Invest Ophthalmol Vis Sci. 2018;59:3920-31.

15. Choi M, Kim J, Kim EK, et al. Comparison of the conventiona Dresden protocol and accelerated protocol with higher ultraviolet intensity in corneal collagen cross-linking for keratoconus. Cornea. 2017;36:523-9.

16. Gore DM, Leucci MT, Koay SY, et al. Accelerated pulsed high-fluence corneal cross-linking for progressive keratoconus. Am J Ophthalmol. 2021;221:9-16.

17. Faramarzi A, Hassanpour K, Rahmani B, et al. Systemic supplemental oxygen therapy during accelerated corneal crosslinking for progressive keratoconus: randomized clinica trial. J Cataract Refract Surg. 2021;47:773-9.

18. Rubinfeld RS, Caruso C, Ostacolo C. Corneal cross-linking: the science beyond the myths and misconceptions. Cornea. the science bey

19. Sachdev GS, Ramamurthy S, Soundariya B, Dandapani R. Comparative analysis of safety and efficacy of topographyguided customized cross-linking and standard cross-linking in the treatment of progressive keratoconus. Cornea. 2021;40:188-93

20. Seiler TG, Fischinger I, Koller T, et al. Customized corneal crosslinking: one-year results. Am J Ophthalmol. 2016;166:14-21.
21. Piñero DP, Alio JL. Intracorneal ring segments in ectatic cornea disease - a review. Clin Exp Ophthalmol. 2010;38:154-67.

22. Colin J, Cochener B, Savary G, Malet F. Correcting keratoconus with intracorneal rings. J Cataract Refract Surg. 2000;26: with intracorr

23. Schanzlin DJ, Asbell PA, Burris TE, Durrie DS. The intrastromal corneal ring segments. Phase $\|$ results for the correction of myopia. Ophthalmology. 1997;104:1067-78.

24. Sharma IP, Bakshi R, Chaudhry M. Corneal collagen crosslinking with and without simultaneous intrastromal corneal rin segment implantation: one-year pilot study. Eur J Ophthalmol. 2021;31:61-68.

25. Hashemi H, Alvani A, Seyedian MA, et al. Appropriate sequence of combined intracorneal ring implantation and corneal collagen cross-linking in keratoconus: a systematic review and meta-analysis. Cornea. 2018;37:1601-7.

26. Hersh PS, Issa R, Greenstein SA. Corneal crosslinking and intracorneal ring segments for keratoconus: a randomized study of concurrent versus sequential surgery. I Cataract Refract Surg. 2019;45:830-9.

27. van Dijk K, Parker J, Tong CM, et al. Midstromal isolated Bowman layer graft for reduction of advanced keratoconus: a Bowman layer graft for reduction of advanced keratoconus: a
technique to postpone penetrating or deep anterior lamellar technique to postpone penetrating or deep anterior
keratoplasty. JAMA Ophthalmol. 2014:132:495-501.

28. van der Star L, van Dijk K, Vasiliauskaite I, et al. Long-term outcomes of Bowman layer inlay transplantation for the treatment of progressive keratoconus. Cornea. 2021. do $10.1097 / \mathrm{ICO} .000000000000287$
29. Alió JL, Alió Del Barrio JL, El Zarif M, et al. Regenerative surgery of the corneal stroma for advanced keratoconus: 1-year outcomes. Am J Ophthalmol. 2019;203:53-68.

30. Alió Del Barrio JL, El Zarif M, de Miguel MP, et al. Cellular therapy with human autologous adipose-derived adult stem cells for with human autologous adipose-derived adult stem
advanced keratoconus. Cornea. 2017:36:952-60.

31. Gore DM, Leucci MT, Anand V, et al. Combined wavefrontguided transepithelial photorefractive keratectomy and corneal crosslinking for visual rehabilitation in moderate keratoconus. $J$ Cataract Refract Surg. 2018;44:571-80.

32. Shah S, Mohan S, Rajan M, et al. Our experience with Athens protocol-simultaneous topo-guided photorefractive keratectomy followed by corneal collagen cross linking for keratoconus. Int J Res Med Sci. 2016;4:2639-44.

33. Grentzelos MA, Liakopoulos DA, Siganos CS, et al. Long-term comparison of combined t-PTK and CXL (Cretan Protocol) versus CXL with mechanical epithelial debridement for keratoconus. J Refract Surg. 2019;35:650-5.

34. Alpins N, Stamatelatos G. Customized photoastigmatic refractive keratectomy using combined topographic and refractive keratecto for ming and refractive data for myopia and astigmatism in eyes with forme fruste and $m$ 2007;33:591-602

35. Song Y, Zhang J, Pan Z. Systematic review and meta-analysis of clinical outcomes of penetrating keratoplasty versus deep anterior lamellar keratoplasty for keratoconus. Exp Clin Transplant. 2020;18:417-28. 